\title{
Max Duttenhofer (1843-1903) und die Anfänge der Rüstungsindustrie im deutschen Südwesten
}

\author{
Von Jörg Kraus
}

Dass in Württemberg und Baden im Zuge der Industriealisierung ein beachtliches Zentrum der Rüstungsindustrie heranwuchs, ist heute kaum noch zu erkennen. Pulver aus Rottweil, Patronen aus Karlsruhe und Waffen aus Oberndorf daraus erwuchsen um 1900 bedeutende Wirtschaftszweige. Für die Kriegsführung ebenso bedeutsam war auch die Erfindung des Automobils, und da die beiden Mitgesellschafter von Gottlieb Daimler aus Rottweil und Karlsruhe kamen, darf man diese Verbindungslinie nach Stuttgart bei einer Betrachtung über die Anfänge der Rüstungsindustrie im deutschen Südwesten hinzunehmen. Bekannt sind in der Regel die Tüftler und Erfinder, insbesondere wenn sie auch im Firmennamen fortleben wie „Mauser“ oder „Daimler“. Weniger bekannt sind mitunter die Unternehmer und das unternehmerische Handeln, das diese Firmen groß werden ließ. Max Duttenhofer aus Rottweil ist ein solcher Unternehmer der Gründerzeit. 1843 geboren, evangelisch, Sohn eines Apothekers, Ausbildung in einer Handelsschule, Apothekerlehre und chemische Zusatzausbildung am Polytechnikum in Stuttgart - das sind schon erste wesentliche Hinweise auf eine Karriere, die schon mit zwanzig Jahren begann. Den jungen Mann zog es nicht in die Apotheke, sondern unmittelbar in die Pulvermühle in Rottweil, in welche sich sein Vater als Teilhaber eingekauft hatte. Und hier war er bald nicht mehr nur ein Pulvermüller, der einen handwerklichen Betrieb führte, sondern ein an neuen wissenschaftlichen und wirtschaftlichen Methoden orientierter Unternehmer.

Der Name Duttenhofer wurde zwar durchaus biographisch bearbeitet ${ }^{1}$, doch erschien er in der Geschichtsschreibung des Landes lange Zeit nur als Randfigur und war selbst in seiner Heimatstadt Rottweil nur den Eingeweihten bekannt. In Rottweil wurde man sich der industriellen Vergangenheit und der architektoni-

${ }^{1}$ Max Cramer, Familie Duttenhofer. Stammbaum und Voreltern, Eßlingen 1901. Otto Schmid, Max Duttenhofer, in: Schwäbische Lebensbilder, Bd.1, hg. von der Württembergischen Kommission für Landesgeschichte, Stuttgart 1940, S.114-124. Paul Genring, Max Wilhelm von Duttenhofer, in: NDB, Bd.4, Berlin 1959, S. 206-207; Onlinefassung: http:// www.deutsche-biographie.de/ppn116258462.html. 
schen Hinterlassenschaft der Pulverfabrik erst nach dem Weggang des Nachfolgebetriebes und über die Auseinandersetzung mit dem Konversionsgebiet bewusst. Das Nylonwerk der Rhodia AG hatte sich schon in den 1980er Jahren schrittweise auf einen Kernbereich des Fabrikareals reduziert und stellte 1994 den Betrieb ein. Bernhard Laule hat aus Sicht des Denkmalschutzes schon 1984 eine Bestandsaufnahme vorgenommen ${ }^{2}$. Nach verschiedenen weiteren Etappen der Aufarbeitung des Gebäudebestandes im Zuge der Neunutzung wurde die architektonische Entwicklung 2012 auch in Buchform publiziert ${ }^{3}$. Eine Darstellung der geschichtlichen Entwicklung der Pulverfabrik fand durch Otto Schmid in zwei kleinen Jubiläumsschriften 1939 und 1940 statt, mit wirtschaftshistorischen Fragestellungen nach der Bedeutung dieses Monopolbetriebes für Rottweil beschäftigte sich jedoch erst Lothar Weisser $1980^{4}$. Als das Ende der industriellen Produktion noch nicht in Sicht war, betrieb Jörg Kraus im Rahmen einer lokalen Ausstellung 1986 eine Recherche $\mathrm{zu}$ den noch vorhandenen Archivalien, die auch Grundlage für die 2001 erschienene geschichtliche Darstellung war ${ }^{5}$.

Überregional fand Max Duttenhofer als erster Vorsitzender der Daimler Motorengesellschaft (DMG) eine gewisse Beachtung, meist jedoch in der Erzählung, wie Daimler gegen seine zwei uneinsichtigen Mitgesellschafter Lorenz und Duttenhofer zu kämpfen hatte. Harry Niemann gelingt es in seinen Darstellungen zu Gottlieb Daimler und Wilhelm Maybach, die Wechselbeziehungen zwischen den Personen zu differenzieren. Doch auch für ihn bleibt es eine Anstrengung, das negativ geprägte Bild Duttenhofers zu relativieren und sowohl die Sichtweise als auch die Rationalitätskriterien der Finanziers einzubeziehen. Er bezeichnet Daimler als Erfinder-Unternehmer, Maybach als Erfinder-Ingenieur, doch für Duttenhofer fehlt eine Kategorisierung ${ }^{6}$. Diese müsste vermutlich Erfinder-Unter-

2 Bernhard Laule, Die ehemalige Pulverfabrik Rottweil am Neckar, in: Denkmalpflege Jg. 13 Nr. 4 (1984) S.124-133.

3 Stefan KIng/Hermann Klos (Hg.), Industriekultur im Neckartal Rottweil. Vom Pulver über Nylon bis zur gewerblichen Vielfalt, Rottweil 2012.

${ }^{4}$ Otto Schmid, Vom braunen prismatischen Pulver zum R.C.P. Aus der Geschichte der Fabrik Rottweil. Ein Gedenkblatt für den Beginn der Großherstellung rauchlosen Nitrocellulose-Blättchen-Pulvers vor 50 Jahren, hg. von der I. G. Farbenindustrie Aktiengesellschaft Rottweil, Rottweil 1939. Otto Schmid, 50 Jahre „Köln-Rottweil“. Aus der Geschichte der Fabrik Rottweil. Die Entstehungsgeschichte und die Leistungen eines bedeutenden Zweiges der chemischen Industrie, hg. von der I. G. Farbenindustrie Aktiengesellschaft Rottweil, Rottweil 1940. Lothar Weisser, Rottweils Wirtschaft und Gesellschaft vom Ende der Reichsstadtzeit bis zum Ersten Weltkrieg (Veröffentlichungen des Stadtarchivs Rottweil, Bd.4), Rottweil 1978.

${ }^{5}$ Jörg Kraus, Für Geld, Kaiser und Vaterland. Max Duttenhofer, Gründer der Rottweiler Pulverfabrik und erster Vorsitzender der Daimler-Motoren-Gesellschaft, Heidelberg 2014.

${ }^{6}$ Harry Niemann, Gottlieb Daimler. Fabriken, Banken und Motoren, Vaihingen/Enz 2000, S. 17. 
Abb. 1: Blick in die Pulvermühle Rottweil (Vorlage: Stadtarchiv Rottweil). [Die Abbildung kann aus rechtlichen Gründen online nicht bereitgestellt werden.]

nehmer-Finanzier lauten, mit einem Schwerpunkt auf „Unternehmer“. Geschäftsanteile gegen Geld und unternehmerisches Know-how, das gilt damals wie heute und führt immer wieder dazu, dass mit jeder Finanzierungsrunde mehr Anteile an den Geldgeber gehen und den eigentlichen Gründern im ungünstigen Fall nur wenig bleibt. Duttenhofer gehört im Gegensatz zu Daimler und Mauser zu jenen Unternehmern, die in diesem Finanzierungsgeschäft nicht untergingen, sondern erfolgreich mitmischten. Der vorliegende Artikel versucht daher den Unternehmer Max Duttenhofer vor der Hintergrundsfolie dieser Finanzierungsgeschäfte, Fusionen und Monopolbildungen nochmals einzuordnen und das Gewicht, das die Rüstungsindustrie im deutschen Südwesten um 1900 erlangte, herauszustellen.

1863 begann der Berufseintritt Duttenhofers in eine klassische Mühle mit Mühlenkanal, Mühlenrad und davon angetriebenen Stampfen zum Mahlen und Vermengen von Salpeter, Kohle und Schwefel zu Schwarzpulver. Aus den Anfangsjahren berichten Eintragungen in sein Notizbüchlein, dass der Küfer wieder zu lange an dem Arsenalfässchen gerichtet habe, die Pulverchargen werden notiert oder es wird vermerkt, dass er seinen sechs Arbeitern zu Weihnachten Schuhe besorgen will (Abb. 1).

Knapp zehn Jahre später, 1872, - der Krieg gegen Frankreich war auch für Duttenhofer erfolgreich, - findet die Umwandlung in die damals neue Form der 
Abb. 2: Max von Duttenhofer (1843-1903) erhielt 1896 den persönlichen Adel. Aufnahme um 1885

(Vorlage: Neuner-Duttenhofer).

[Die Abbildung kann aus rechtlichen Gründen online nicht bereitgestellt werden.]
Aktiengesellschaft statt. Max Duttenhofer war nun Vorstand und Direktor der „Pulverfabrik Rottweil“. Da er gleichzeitig Hauptaktionär war, hatte sich nichts Wesentliches geändert, doch konnte nun die Expansion weiter vorangetrieben werden. In rascher Folge wurden andere Pulvermühlen aufgekauft und ein System von Pulvermagazinen errichtet, um den Handel $\mathrm{zu}$ erleichtern. Im Geschäftsbericht 1873/1874 sind nun 20 Arbeiter und 5 Beamte vermerkt (Abb.2).

Schon 1877 musste durch die Einrichtung eines Zweigwerkes in Geesthacht bei Hamburg der Name in „Pulverfabriken Rottweil-Hamburg“ erweitert werden. Dieses Zweigwerk trug ganz wesentlich zum Aufschwung der Fabrik bei, denn von Hamburg aus gediehen die Exportgeschäfte und die Marine konnte mit schwerem Geschützpulver beliefert werden. Während von Rottweil aus vor allem die Balkanstaaten beliefert wurden, konnten von Hamburg aus die Geschäftsbeziehungen in die ganze Welt aufgebaut werden. Das Werk wurde geleitet vom Bruder Carl Duttenhofer. Und es befand sich in Nachbarschaft zur Dynamitfabrik Krümmel von Alfred

Nobel, die schon 1865 in Geestacht entstanden war.

Solche Nachbarschaften waren sicher günstig. Besonders günstig war aber wohl die Nachbarschaft zu Reichskanzler Bismarck, denn das Fabrikgelände bei Geesthacht gehörte dem Fürsten Bismarck und wurde von diesem gepachtet. Bismarck selbst gab der neuen Pulverfabrik den Namen „Düneberg“. In Max Duttenhofer hatte Bismarck nicht nur einen patriotischen Schwaben und treuen Anhänger gefunden, sondern auch einen Pulverproduzenten, den er zur Steigerung der Leistungsfähigkeit des deutschen Heeres protegierte. Angeheizt durch die politische Auseinandersetzung mit Frankreich und eine latente Kriegsangst, war ein Klima des Wettrüstens entstanden, welches dem Geschäft Duttenhofers recht förderlich war. In enger Zusammenarbeit mit der Firma Krupp wurden 
ständige Verbesserungen an den Geschützen und den zugehörigen Pulvern vorgenommen. Ende der 70er Jahre wurde so das „braune prismatische Pulver“ entwickelt, das vor allem für schwere Geschütze hervorragende Eigenschaften besaß. Über die weltweiten Geschäftskontakte von Krupp wurden nun auch Pulveraufträge vermittelt.

Ein entscheidender Entwicklungssprung folgte dann mit der Entwicklung eines chemischen Pulvers. Von verschiedenen Seiten wurde daran gearbeitet, doch Duttenhofer gelang es Anfang der 1880er Jahre als Erstem in Deutschland, ein solches Pulver herzustellen. Zellulosebestandteile waren schon beim braunen prismatischen Pulver notwendig, nun kam ein Nitrierungsprozess hinzu und führte zu Nitrozellulose als Ausgangsstoff. Viele Sprengstoffe sind Nitroverbindungen. Nitrate sind die Salze und Ester der Salpetersäure. Nitrate sind allgegenwärtig und dienen als Quelle für Stickstoffverbindungen und sind Nährstoffe für die Pflanzen. Abbauwürdige Mengen von Nitraten finden sich hauptsächlich in Chile, daher bezog die Pulverindustrie von dort diese Salze unter dem gängigen Namen Chilesalpeter. Aus den chemischen Verwandschaftsbeziehungen ergeben sich die Beziehungen zwischen den industriellen Anwendungen, die vom Schwarzpulver über das chemische Pulver bis zum Nitroglycerin von Alfred Nobel reichen und auch begründen, weshalb das Haber-Bosch-Verfahren zur Ammoniaksynthese (Stickstoff und Wasserstoff) im ersten Weltkrieg die Pulver- und Sprengstoffherstellung aufrecht erhalten konnte und gleichzeitig zur Kunstdüngerproduktion essentiell wurde. Aus den chemischen Verwandtschaftsverhältnissen nitrierter Zellulose ergab sich später auch die Nähe zwischen den Herstellungsverfahren von Pulver, Viskosefasern (Celluloseregeneratfasern) und der Filmherstellung (Zelluloid). All diese verwandten chemischen Anwendungen fanden sich daher in den zwanziger Jahren vereint unter dem Dach der IG Farben AG. Dies macht deutlich, an welcher Nahtstelle auch Duttenhofer im Jahr 1884 stand, als er sein chemisches Pulver bei Schießversuchen einer Kommission des Kriegsministeriums vorstellte. Es war eine jener epochalen technischen Veränderungen, die zum Aufbau neuer Industriezweige führten, aber natürlich auch zu einer veränderten Kriegsführung. Er war sich der Bedeutung seiner Erfindung sicherlich bewusst, denn er hatte für dieses Ereignis eigens einen Fotografen bestellt, der den historischen Moment festhalten sollte (Abb.3).

Dieses chemische Pulver hatte zwar noch Schwächen, doch Reichskanzler Bismarck wollte es um jeden Preis und setzte gegen den massiven Widerstand seines Kriegsministers durch, dass das Pulver angeschafft wurde. Der brieflich ausgetragene Streit zwischen Bismarck und seinem Minister Bronsart von Schellendorf geht so weit, dass dieser ihm sogar den Rücktritt anbot. Die Auseinandersetzung liest sich fast wie ein Polit- und Wirtschaftskrimi. Bismarck war bewusst, dass Kriege v.a. mit Innovationen zu gewinnen waren und ein rauchfreies chemisches Pulver war genau eine solche Innovation. Er setzte gegen den Rat seines Ministers einen Vertrag durch und Duttenhofer konnte mit dem Geld des Kriegsministe- 
Abb. 3: Schießversuch mit dem Rottweiler Chemischen Pulver (R. C.P.). In der Mitte hinter den Schützen Max Duttenhofer, links von ihm Hauptmann v. Sack (mit Vollbart) von der Gewehrprüfungskommission aus Spandau. Aufnahme vom Oktober 1884 (Vorlage: Stadtarchiv Rottweil). [Die Abbildung kann aus rechtlichen Gründen online nicht bereitgestellt werden.]

riums seine Fabrikanlagen so weit ausbauen, dass er gegen Ende der 1880 er Jahre endgültig zu den Großen im Pulvergeschäft gehörte ${ }^{7}$.

Was bedeutete dies? In Rottweil war die Pulverfabrik mit Abstand der größte Betrieb mit 854 Arbeitsplätzen (1890) und der Einfluss Duttenhofers in der Stadt war in allen Bereichen spürbar. Eine breite Industrialisierung erwuchs daraus dennoch nicht, Rottweil entwickelte sich eher in Richtung einer Beamtenstadt und stand der klassischen Industriearbeit distanziert gegenüber. Diese war insbesondere in der Pulverfabrik natürlich auch nicht gerade erstrebenswert, da mit hohen Gefahren verbunden. Regelmäßig kam es zu Explosionen und die Arbeit mit den Säuren führte zu erheblichen gesundheitlichen Gefährdungen ${ }^{8}$ (Abb. 4).

7 Das Rottweiler Chemische Pulver RCP wurde sehr schnell durch bessere Entwicklungen überholt, doch der Vertrag blieb bestehen und die Fabrikation auf verbesserte Herstellungsmethoden umgerüstet.

${ }^{8}$ Gustav Martin, Geschichtliche Entwicklung der Kartellbildung in der deutschen Sprengstoff-Industrie, Diss. Heidelberg 1903, S.131 stellt eine Statistik für die Pulverindustrie in der Zeit zwischen 1891 und 1900 zusammen, nach welcher jährlich zwischen 65 und 95 Fälle mit über 13-wöchiger Erwerbsunfähigkeit aufgeführt sind sowie jährlich zwischen 10 und 44 Todesfälle, jeweils bezogen auf 10.000 Arbeiter. 


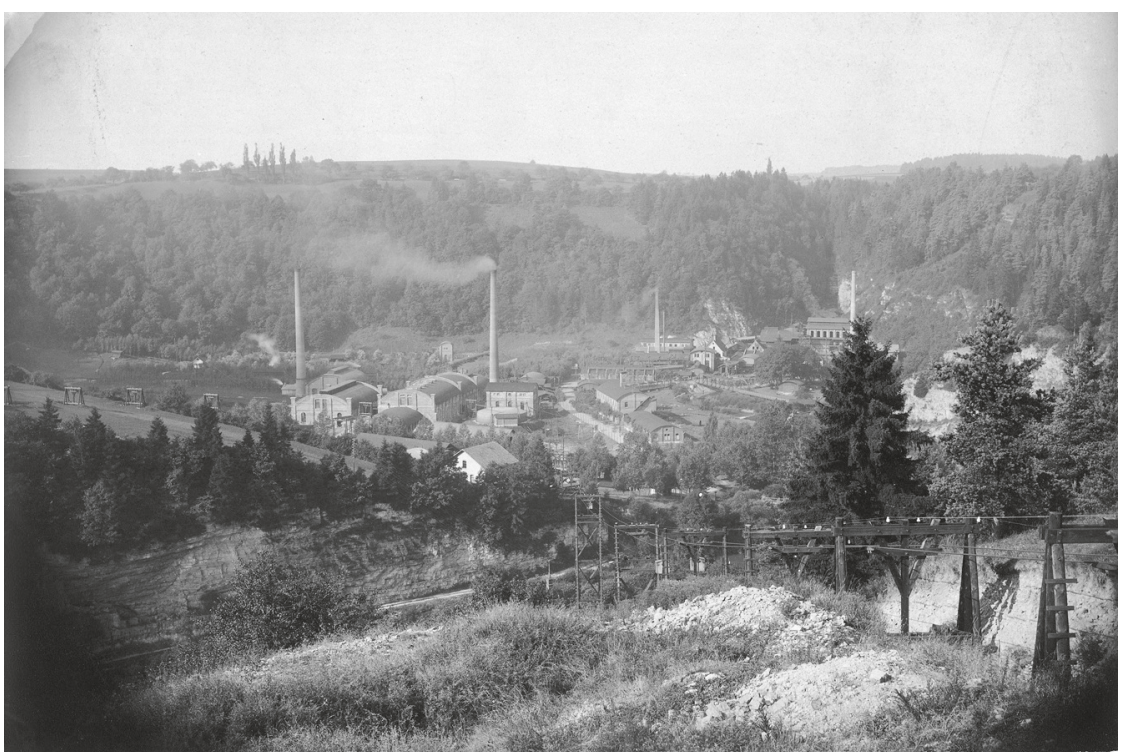

Abb. 4: Das Rottweiler Fabrikgelände in einer Schlaufe des Neckartals in der Ausbauform nach 1890 (Vorlage: Verfasser).

Ökonomisch bedeutete der Auftrag vom Kriegsministerium den kompletten Um- und Ausbau der Fabrik. Das Gelände war über einen Betriebsbahnhof versorgt und hatte zum internen Materialtransport $2.700 \mathrm{~m}$ Drahtseilhängebahnen. Das Grundkapital der Firma lag 1872 noch bei 210.000 Mark, stieg mit der Einrichtung der Pulverfabrik Rottweil-Hamburg auf 600.000 Mark und lag 1884 schon bei 2,7 Millionen. Von da an folgten Fusionen und Kartellbildungen in diverse Richtungen. Aus dem Zusammenschluss mit den Vereinigten Rheinisch-Westfälischen Pulverfabriken gingen 1890 die „Vereinigten Köln-Rottweiler Pulverfabriken“ mit einem Grundkapital von 16,5 Millionen Mark hervor. Zusammen mit zwei weiteren bereits verbundenen Pulverfabriken wurde ein „Generalkartell“ mit neun Firmen der „Sprengstoffgruppe“ gebildet. Damit war insbesondere zum Nobel Dynamit Trust eine engere geschäftliche Verbindung hergestellt. Dass diese bald sehr eng war, zeigt ein Bericht der Vereinigten Köln-Rottweiler Pulverfabriken von 1893: „In der Herstellung von Nitrocellulose führt ein rückhaltloser Austausch der beiderseitigen reichen Erfahrungen zu schätzenswerten Fortschritten und Verbesserungen, während die Verarbeitungen dieser Rohmaterialien zu Pulver unsere Erfahrung zur besten und erfolgreichsten Verwendung brachte. Dazu ermöglicht die nachbarschaftliche Lage der Dynamitfabrik Krümmel zu unserer Pulverfabrik Düneberg ein ökonomisches Hand in Handarbeiten. Hervorzuheben ist, 


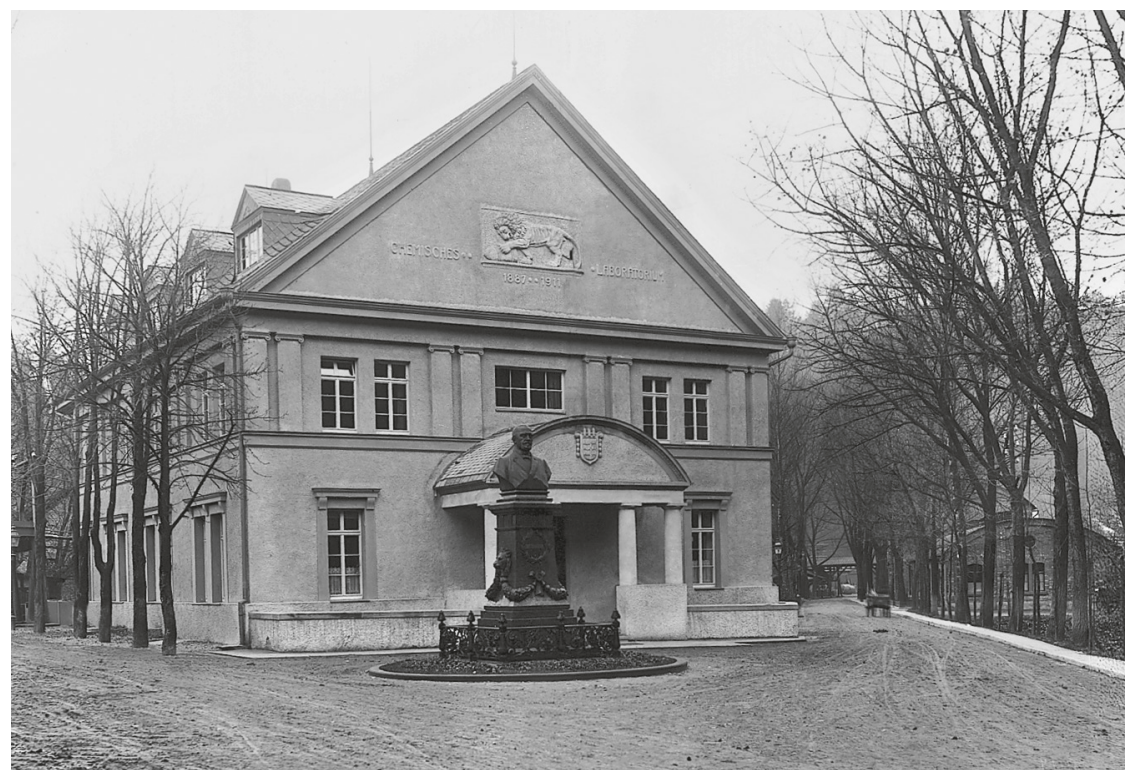

Abb. 5: Das Chemische Laboratorium mit dem Denkmal Duttenhofers, 1910/1911 im Rottweiler Werk am Werkseingang erbaut. Im Giebelrelief spielt ein Löwe mit einer Granate, darunter das württembergische Wappen, nicht durch eine Krone, sondern durch drei Granaten geschmückt (Vorlage: Verfasser).

dass neue Erfindung, z.B. Amidpulver, und mit ihr verknüpfte entsprechende Neueinrichtung der Werke, nur von Kapitalkräftigen realisiert werden können“9. In die gleiche Zeit fiel die Ausweitung des Geschäftes in Richtung Patronen und Gewehre, sowie der Einstieg Duttenhofers als Gesellschafter bei der Motorenfabrikation von Gottlieb Daimler.

Laufende technische Innovationen und ein damit einhergehender großer Kapitalbedarf prägten diese Zeit. Es zeigt sich, dass die Kombination von beidem nicht jedem Firmengründer gleichermaßen glückte, Duttenhofer jedoch in beiden Feldern erfolgreich war. Der Übergang vom Schwarzpulver zum chemischen Pulver war ohne Wissenschaftler bald nicht mehr denkbar. Aus seinem kleinen Laboratorium entwickelte sich sehr schnell ein zentraler Betrieb mit Chemikern, die jede Pulvercharge genau zu kontrollieren hatten. Dass eine solche wissenschaftliche

\footnotetext{
${ }^{9}$ Martin (wie Anm. 8) S. 94.
} 
Grundlage auch für die Weiterentwicklung immer notwendig sein würde, lag für Duttenhofer aufgrund seiner eigenen Erfahrungen auf der Hand. Er selbst war dann auch recht stolz darauf dass es ihm gelang, 1898 die Zentralstelle für Wissenschaftlich-Technische Untersuchungen in Neubabelsberg ins Leben zu rufen und deren Finanzierung durch die verbundenden Unternehmen sicher zu stellen. Die experimentellen Arbeiten der Zentralstelle hatten vielfach sehr praktische Anwendungsgebiete. So ging es z.B. darum, nicht alle Nitroverbindungen pauschal als Sprengstoffe einzustufen und damit Einfluss auf die Deutsche Eisenbahnverkehrsordnung zu nehmen. Der Transport war sowohl für die Farbenindustrie als auch für die Pulverindustrie von hoher Bedeutung ${ }^{10}$ (Abb.5).

\section{Gründerzeit ist Pulverzeit}

Halten wir an dieser Stelle zunächst fest: Gründerzeit ist auch Pulverzeit! Aus dem Pulverdampf der Kriege entstehen nicht nur die Nationalstaaten sondern auch mächtige Industriezweige. Insbesondere die ständigen Unruheherde auf dem Balkan sowie das im Niedergang begriffene Osmanische Reich boten reichlich Absatzmärkte. Nicht von ungefähr hatte auch der Erste Weltkrieg seinen Auslöser auf dem Balkan. Von Rottweil aus wurden all diese Staaten beliefert und in Rottweil hatte man sich schon an die fremdländischen Gäste gewöhnt, die regelmäßig in der Villa Duttenhofer zu Gast waren.

Das nationale Pathos nach dem Krieg 1871/1872, die Auflösung der Zollschranken 1872, der Ausbau der Transportwege mit der Eisenbahn, neue Finanzierungsmöglichkeiten über die Aktiengesellschaft - all diese Faktoren befeuerten den Aufbau neuer Wirtschaftsstrukturen. Ein durchsetzungswilliger Mann wie Duttenhofer, von dem seine Enkelin berichtet, dass er mit seinen Vierspännern so waghalsig fuhr, dass er immer wieder verunglückte, passte gut in diese Zeit. Wie mit den Gespannen, so hielt er es offenbar auch mit seinen Investitionen. Die Enkelin gibt auch die Erzählung ihrer Großmutter wieder, nach der [...] Großvater für damalige Zeiten enorme Einnabmen gehabt hätte, er hätte mitunter im Jabr 13 Millionen verdient, aber allerdings im nächsten vielleicht 12 Millionen verloren, weil er immer wieder Nenes anfing, experimentierte und einer Sache, Arbeit, Erfindung, Fabrik, sebr schnell überdrüssig wurde, sobald sie florierte, lief und Einnabmen brachte. Ihn reizte es mehr, Schwierigkeiten zu überwinden ${ }^{11}$.

${ }^{10}$ Heinrich Brunswig, Die Zentralstelle für wissenschaftlich-technische Untersuchungen in Neubabelsberg. Zur 25. Wiederkehr ihres Gründungstages, in: Zeitschrift für angewandte Chemie 37/38 (1923) S.255-257, hier S. 257.

${ }^{11}$ Unveröffentlichte Erinnerungen von Elisabeth Rönckendorff, Privatbesitz Bernd Neuner-Duttenhofer, S. 55, wiedergegeben bei Kraus (wie Anm.5) S. 86. 
Mit seiner deutsch-nationalen Gesinnung ${ }^{12}$ und mit seinem Firmengelände in Geesthacht bei Hamburg auch Nachbar von Fürst Bismarck, befand sich Duttenhofer politisch in guter Gesellschaft. Er war in unzähligen Gremien und Aufsichtsräten und hatte damit natürlich auch Umgang mit den wichtigen Akteuren des Wirtschaftslebens. 1896 erhielt er den württembergischen Personaladel und wurde damit zum Geheimen Kommerzienrat Max von Duttenhofer. Entsprechend standesgemäß wurden seine beiden Töchter Anna und Elsa auch verheiratet. Beide Ehemänner machten eine klassische Militärkarriere, Alfred von Mohn war schließlich Generalleutnant in Württemberg, Otto von Haldenwang war Oberstleutnant. Und das dritte Kind, der Sohn Max, trat später dann die Nachfolge des Vaters in der Firma an.

Neben allem Patriotismus ging es aber natürlich ums Geld. Und dieses wurde gerade mit Hilfe der Kartellverbände sehr gut verdient. Da im Falle des Militärpulvers allerdings nur der Staat als Abnehmer auftrat, hatte eben dieser gepriesene Nationalstaat das Nachsehen.

Der sozialdemokratisch orientierte „Beobachter“ aus Stuttgart geht in einem Zeitungsbericht vom 22. März 1894 ausführlich auf die Kartellbildung ein und schreibt: „[...] dieser Ring erblickt seine vornehmste Aufgabe darin, die Militärpulverpreise auf einer ganz ungewöhnlichen Höhe zu erhalten“. Der Autor erkennt den Pulverfabrikanten Max Duttenhofer an der Spitze der Bewegung und zählt die Namen der mitwirkenden Akteure vom Bankier Dr. Kilian Steiner über Alfred Nobel in Paris, Ludwig Loewe in Berlin und Friedrich Krupp in Essen auf. „Wir erblicken da eine stolze imposante Gesellschaft von Kanonen-, Flinten-, Pulver- und Dynamitkönigen [...]“ und: „[...] der Pulverring hat Reingewinn-Ergebnisse von 100-150\% zu verzeichnen [....]. Nun wird im Artikel vorgerechnet, dass allein von den Fabriken Rottweil und Düneberg in den Jahren 1891 bis 1893 ca. 25 Millionen Mark an den Staat verkauft wurden und der überhöhte Preis sicherlich 3 Millionen Mark ausgemacht habe. „Der Vorsitzende des Aufsichtsrates, Geheimer Kommerzienrat Duttenhofer, erhält $5 \%$ der Verkaufssumme aus dem in Rottweil, und $1 \%$ aus dem in Düneberg gefertigten rauchlosen Militärpulver. Das entspricht 1890 bis 1893 einer Summe von etwa 750.000 Mark. Dazu kommt noch sein Anteil an Tantiemen des Aufsichtsrates und der Dividenden-Ertrag aus seinem sehr beträchtlichen Aktienbesitz ${ }^{13}$.

12 Duttenhofer war Mitglied der Deutschen Partei und trat auch dem Alldeutschen Verband bei, der 1891 gegründet wurde und der auch Bismarck als Mitglied gewinnen konnte. Alldeutsch meinte die Gemeinschaft aller Deutschen und machte damit nicht an den reichsdeutschen Grenzen halt, sondern proklamierte die Ausweitung nach Osten, die Entfaltung der germanischen Rasse und ähnliche Ideen, die später dann deutsche Geschichte prägen sollten. Vgl. https://de.wikipedia.org/wiki/Kategorie:Mitglied_im_Alldeutschen_Verband (20.8.2015).

${ }^{13}$ Der Beobachter. Ein Volksblatt aus Württemberg, Stuttgart, 22. März 1894. In einer Übersicht stellt Gustav Martin die Dividenden für die Pulverfabrik Rottweil-Hamburg für 
Sehen wir über alle Ungenauigkeiten solcher Berechnungen hinweg, so ist ein Jahreseinkommen zwischen einer und den von der Enkelin erwähnten 13 Millionen sicherlich zu erwarten. Die Gründerzeit brachte gerade in Baden-Württemberg eine Reihe von Industriezweigen zur Blüte und legte damit eine industrielle Basis für das Land, sowie eine pekuniäre Grundlage für eine neue Klasse an Millionären. Eine Aufstellung der Millionäre in Baden-Württemberg aus dem Jahr 1914 - und damit schon elf Jahre nach dem Tod Max von Duttenhofers 1903 - listet die Familien der drei Kinder Anna, Elsa und Max mit einem Vermögen von jeweils 5 Millionen Mark auf. Willi Boelke, der diese Aufstellung von 1914 herausgibt und kommentiert, schätzt, dass Max von Duttenhofer ungefähr 15 Millionen Mark vererbt hat und vergleicht dies mit dem Kollegen Alfred Nobel, der ein Vermögen von 32 Millionen Schweden-Kronen für seine Stiftung hinterlassen hatte ${ }^{14}$. Dies wären nach damaligem Umrechnungskurs ca. 36 Millionen Mark gewesen, mithin konnte Nobel etwas mehr als das Doppelte des Duttenhofer'schen Erbes hinterlassen ${ }^{15}$.

Der durchschnittliche Jahreslohn für Arbeiter in der Explosivstoff-Industrie lag im Jahr 1900 bei 1.050 Mark $^{16}$ und aufgrund der Gefährlichkeit der Arbeit eher über dem Durchschnitt anderer Branchen. Verdienste in Staat und Wirtschaft, die über 10.000 Mark Jahresgehalt hinausgingen, waren in dieser Zeit also schon hoch. Rudolf Martin, der die Zusammenstellung der württembergischen Millionäre z.T. aus Quellen und z.T. aus Rückschlüssen vornahm, stellt einen Zusammenhang zwischen den 520 Personen mit einem Jahreseinkommen von über 60.000 Mark mit den von ihm geschätzten 560 Vermögensmillionären her ${ }^{17}$. Nicht ganz an der Spitze aber doch sehr weit oben in der Aufstellung findet sich auch seine Majestät, König Wilhelm II. von Württemberg, mit einem Jahreseinkommen von 2,9 Millionen und einem Vermögen von 36 Millionen Mark.

Dies lässt ungefähr die Relationen ermessen und zeigt, dass das Einkommen der Nachkommen Duttenhofers immer noch zu den höchsten im Lande gehörte. Der Sohn Dr. Max Duttenhofer, der seinem Vater nachfolgte und 1908 einer der

die Jahre 1872 bis 1889 zusammen, die im Durchschnitt zwischen 15 und $20 \%$ lagen; MarTin (wie Anm. 8) S.66. Paul Siebertz ergänzt, dass nach den öffentlichen Geschäftsberichten die Vereinigten Köln-Rottweiler Pulverfabriken in den Jahren 1890/1893 in der Lage waren, an ihre Aktionäre Dividenden von insgesamt 8.433.000 Mark und an die Aufsichtsräte Tantiemen von zusammen 1.083.508 Mark auszuschütten; Paul SieberTz, Gottlieb Daimler. Ein Revolutionär der Technik, München/Berlin 1940, S. 204.

${ }^{14}$ Willi A. Boelcke, Millionäre in Württemberg. Herkunft - Aufstieg - Traditionen. Mit dem Faksimile-Abdruck des „Jahrbuchs des Vermögens und Einkommens der Millionäre in Württemberg und Hohenzollern“ von Rudolf Martin 1914, Stuttgart 1997, S. 32.

15 Umrechnungskurs: ca. 89 Kronen = 100 Reichsmark in den Jahren zwischen 1900 und 1914. Vgl. die Liste der historischen Wechselkurse bei der Schwedischen Reichsbank: http:// www.riksbank.se/sv/Riksbanken/Forskning/Historisk-monetar-statistik/Valutakurser/ Valutakurser-i-Sverige-18001914/ (20.8.2015).

16 Martin (wie Anm. 8) S. 130.

17 Boelcke (wie Anm. 14) S. 105. 
Generaldirektoren der Köln-Rottweiler Pulverfabriken wurde, wird mit einem jährlichen Einkommen von 600.000 Mark angegeben und gehört damit laut Martin zu einem Kreis von nur noch 10 Personen mit Einkommen in dieser Höhe oder darüber ${ }^{18}$. In gleicher Höhe lag auch das Einkommen von Carl Duttenhofer, dem jüngeren Bruder des Max von Duttenhofer. Auch dieser war Generaldirektor und damit vornehmlich in Berlin tätig. Boelcke konstatiert: „Die weitaus größte Kapitalzusammenballung in den Händen württembergischer Industrieller verband sich noch vor Bosch mit dem Namen der Familie Duttenhofer in Rottweil und der raschen Expansion ihrer Pulverfabrik zum Pulverkonzern und damit zur ersten württembergischen Weltfirma, wie der ,Schwäbische Merkur' 1903 lobend hervorhob"19. Freilich setzte sich Robert Bosch ab 1905 mit dem weltweiten Erfolg der Magnetzünder und anderer Entwicklungen in der aufkommenden Automobilindustrie auch in der Einkommenshierarchie ab. Für 1914 wird sein Jahreseinkommen auf 4 Millionen geschätzt. Während Bosch jedoch in Württemberg blieb, wurde die Konzernzentrale der Köln-Rottweiler Pulverfabriken nach Berlin verlagert und daher erfuhr dieser Industriezweig in Baden-Württemberg nach der Gründerzeit keine wesentliche Ausweitung mehr. Natürlich wuchs das Werk Rottweil in den beiden Weltkriegen jeweils auf über 2.000 Beschäftigte an, doch es schrumpfte danach auch wieder.

\section{Die Verbindung zwischen Pulver, Patronen und Gewehren}

Aus dem Kreis der steinreichen Millionäre müssen noch zwei Namen erwähnt werden, die maßgeblich am Aufbau der Finanzbranche beteiligt waren und damit auch an entscheidenden Schalthebeln in der Wirtschaft saßen. Der ältere Dr. Kilian von Steiner, der wie Max von Duttenhofer 1903 starb und ein großes Vermögen vererbte, sowie der jüngere Alfred von Kaulla, der mit einem Einkommen von jährlich 1,1 Millionen Mark zu den absoluten Topverdienern in Württemberg gehörte ${ }^{20}$. Steiner war Ideengeber und maßgeblich am Aufbau der Württembergischen Vereinsbank beteiligt, die als Zielsetzung vor allem die Kapitalisierung der Industrie hatte. Alfred Kaulla wurde dabei sein enger Vertrauter. Beide waren treibende Kräfte bei der Umwandlung der Betriebe in Aktiengesellschaften, bei ihrer Sanierung, bei der Verschmelzung zu größeren Wirtschaftseinheiten sowie beim

18 Ebd. Gemäß seiner Zusammenstellung müsste dieser Kreis bei 30 Personen sein. Offenbar hat Martin bei der Nennung von 10 Personen ein Selektionskriterium gewählt, aber nicht benannt. Der Eintrag zu Duttenhofer ebd., S.121.

19 Ebd., S. 30.

20 Kilian Steiner (1833-1903) erhielt 1895 den württembergischen Personaladel, Alfred Kaulla (1852-1924) erhielt diesen 1893. Ausführlich zum Wirken Steiners vgl. Otto K. Deutelmoser, Kilian Steiner und die Württembergische Vereinsbank (Stuttgarter historische Studien zur Landes- und Wirtschaftsgeschichte, Bd.4), Ostfildern 2003. 
Aufbau von Kartellen und Interessengemeinschaften. Kilian Steiner und die Württembergische Vereinsbank waren ebenso an der Gründung der Deutschen Bank beteiligt. Steiner unterhielt ein freundschaftliches Verhältnis und war Ratgeber für Georg von Siemens. Die Württembergische Vereinsbank ging schließlich 1926 in die Deutsche Bank über und mit ihr auch die intensiven Beziehungen zur Daimler-Motoren-Gesellschaft.

Aber zunächst zurück zur Rüstungsindustrie. Wie alle anderen europäischen Großmächte, so wollte auch das Osmanische Reich ein modernes Repetiergewehr und entschied sich nach recht kurzer Erprobungszeit 1887 für ein Modell der Firma Mauser in Oberndorf. Die Verhandlungen dazu hatten zunächst mit Paul Mauser begonnen, wurden dann jedoch von Alfred von Kaulla übernommen, der von Seiten der Württembergischen Vereinsbank für den kaufmännischen Bereich bei den Mauserwerken eingesetzt worden war. Es lag auf der Hand, dass die Türkei nicht nur Gewehre benötigte und so wurde im Hintergrund alsbald ein Konsortium geschmiedet, das geeignet war, Gewehre, Patronen und Pulver zu verkaufen. Neben Pulver aus Rottweil und Gewehren aus Oberndorf war mit der Metallpatronenfabrik von Wilhelm Lorenz in Karlsruhe grundsätzlich auch die dritte Komponente in der Region verfügbar. Diese Komponenten zusammenzubringen und damit die „Kleinkaliberfrage“ für ganze Heere lösen zu können, versprach glänzende Geschäftsaussichten. Schon im November 1887 teilten Max Duttenhofer und Isidor Loewe ${ }^{21}$ aus Berlin das Geschäftsfeld vertraglich untereinander auf und schrieben eine Fusionsdynamik fest, die in den folgenden Jahren sehr konsequent umgesetzt wurde ${ }^{22}$.

Die Absprachen zwischen Duttenhofer, der Württembergischen Vereinsbank mit Steiner und Kaulla sowie dem Maschinen- und Waffenhersteller Isidor Loewe in Berlin führten letztlich dazu, dass sowohl die Firma Mauser AG als auch die Metallpatronen AG von Lorenz aufgekauft wurden und über Zwischenschritte in die 1896 gegründete Deutsche Waffen- und Munitionsfabriken AG (DWM) eingebracht wurden. Insbesondere bei Wilhelm Lorenz musste ein entsprechender Druck aufgebaut werden, um ihn zum Verkauf zu bewegen ${ }^{23}$.

${ }^{21}$ Die Ludwig Loewe \& Co. AG wurde von Ludwig Loewe bis zu seinem Tod 1886 aufgebaut und danach von seinem Bruder Isidor weitergeführt. Vgl. Hans Christoph Graf von SeHERr-Thoss, Ludwig Loewe, in: NDB, Bd. 15 Berlin 1987, S.77-78; Onlinefassung: http://www.deutsche-biographie.de/ppn117166839.html.

22 Der Vertrag vom 21.11.1887 wurde zwischen der „Ludwig Loewe Commanditgesellschaft auf Actien zu Berlin und dem Herrn Commerzienrath Duttenhofer für sich und die Pulverfabrik Rottweil-Hamburg“ abgeschlossen (Rottweiler Stadtarchiv, Akten der Pulverfabrik Rottweil, lfd. Nr.119). Hierin wurden - ohne dass man schon im Besitz der entsprechenden Firmen gewesen wäre - die verschiedenen Modalitäten zur Verteilung der Lizenz-Gebühren aus den Patenten der Firma Mauser und des Mieg'schen Konsortiums sowie der Erträge aus dem Kleinkaliber-Gewehr und dem dazu gehörigen Pulver geregelt.

${ }^{23}$ Vgl. Kraus (wie Anm. 5) S. 69. 
Aber das gewünschte Resultat stellte sich ein: Nach den erfolgten Fusionsrunden war die DWM ein gewichtiger Faktor in der deutschen Rüstungswirtschaft. Im Aufsichtsrat saßen Duttenhofer und seine Kollegen, Isidor Loewe aus Berlin sowie die erfolgreichen Strategen der Württembergischen Vereinsbank. In der DWM sehen wir einen Kulminationspunkt mehrerer Entwicklungen, die in Württemberg und Baden ihren Ausgangspunkt hatten und zu Schwergewichten im Rüstungsgeschäft führten. Die Einzelbestandteilen Pulver, Patronen und Gewehre wurden mehr und mehr aufeinander abgestimmt, so dass man im Laufe der Verhandlung mit dem Osmanischen Reich zu der Auffassung kam, dass es geschickter wäre, ein technisches Gesamtsystem zu verkaufen. Hier sehen wir also technische Entwicklungen, die ganz entscheidend aus Rottweil, Oberndorf und Karlsruhe kamen, die aber auch aus Berlin nochmals wichtige Impulse erhielten. Denn ein wesentliches Merkmal der Ludwig Loewe \& Co. AG war die Fähigkeit zur Massenproduktion. Ludwig Loewe hatte dies zunächst mit der Fabrikation von Nähmaschinen erprobt, bevor er in die Maschinen- und Waffenproduktion wechselte. Erst die absolute Gleichförmigkeit ließ einen rationellen Herstellungsprozess, aber auch die Reparatur mit Ersatzteilen zu. Die technologische Schrittmacherrolle der Rüstungsindustrie lag daher nicht allein in technischen Innovationen begründet, sondern auch in der Produktionsweise. „Kaum ein Produkt für den zivilen Bedarf wurde damals in so großen Stückzahlen identischer Exemplare hergestellt wie Waffen und militärische Ausrüstung, da ein Markt für Massenprodukte noch nicht existierte “24. Wichtige metallurgische Erkenntnisse entstanden also bei der Entwicklung von Geschützrohren bei Krupp, neue Patronen und Gewehre wurden entwickelt, die chemische Industrie entstand $\mathrm{zu}$ einem wichtigen Teil rund um explosive Nitroverbindungen und die Motorentwicklung ermöglichte nach der Eisenbahn eine neue Mobilität zu Land, $\mathrm{zu}$ Wasser und in der Luft. Damit sind gleichzeitig auch die wesentlichen technischen Voraussetzungen benannt, die schließlich im Ersten Weltkrieg zum Einsatz kamen. Die extremen Gewinnsteigerungen der Köln-Rottweiler Pulverfabriken, der Deutschen Waffen- und Munitionsfabriken wie auch der Daimler Motorengesellschaft im Krieg zeigen, welchen Entwicklungsweg diese im deutschen Südwesten entstandenen Firmen genommen haben ${ }^{25}$. Während des Krieges wurde

${ }^{24}$ Heinrich Walle, Die Auswirkungen des technischen Fortschritts 1871 bis 1914, in: Karl-Volker Neugebauer (Hg.), Grundzüge der deutschen Militärgeschichte, Bd.1, Freiburg 1993, S. 199.

${ }^{25}$ Bontrup und Zdrowomyslaw liefern ein Schaubild zur Gewinnsteigerung der genannten Rüstungskonzerne (S.174) und erwähnen die Diskussion zu überhöhten Gewinnen bei der Firma Daimler (S. 159): Heinz-J. Bontrup/Norbert Zdrowomyslaw, Die deutsche Rüstungsindustrie. Vom Kaiserreich bis zur Bundesrepublik. Ein Handbuch, Heilbronn 1988. Die Dividende der Köln-Rottweiler Pulverfabriken betrug 1913: 20 \%; 1914: 25 \%; 1915: 33 \%; 1916: 20 \%; 1917: 20 \%; 1918: 18 \%. Angaben nach Rudolf FucHs, Die Kriegsgewinne der verschiedenen Wirtschaftszweige in den einzelnen Staaten an Hand statistischer Daten dargestellt, Diss. Zürich 1918, S. 90. 
von Rottweil aus zeitweise der ganze Infanteriepulverbedarf Deutschlands gedeckt, immer aber mindestens $80 \%$ desselben ${ }^{26}$.

All dies wäre ohne die Finanzarchitektur im Hintergrund jedoch nicht denkbar, daher ist der Stellenwert der Württembergischen Vereinsbank in Stuttgart in diesem Zusammenhang kaum hoch genug zu bewerten. Heute würde man sie als die Spezialisten in „Merger and Akquisition“ bezeichnen. Mit den Namen Duttenhofer (Rottweil), Mauser (Oberndorf) und Lorenz (Karlsruhe) sowie Steiner und Kaulla (Stuttgart) sind damit auch wesentliche Akteure benannt. Aus Berlin kommt die Firma Ludwig Loewe \& Co. mit den Brüdern Ludwig und Isidor hinzu, die zunächst in der Rüstungsindustrie und dann auch in der Automobilindustrie auftaucht. Aus Stuttgart ist daher auch noch die Verbindung zu Gottlieb Daimler zu schildern, um den Kreis der Wechselbeziehungen zu schließen. Betrachtet man das Jahrzehnt zwischen 1890 und 1900 dann stellt man hier eine Verschiebung der Machtverhältnisse zwischen diesen Akteuren fest. Insbesondere Mauser und Daimler, die uns als Markennamen erhalten blieben, wurden als Unternehmer entmachtet und jene Namen, die uns heute kaum noch bekannt sind, waren für die unternehmerische Entwicklung deutlich zentraler. Während die einen den Topos des schwäbischen Tüftlers und Erfinders bedienen und v.a. in der Beziehung zur Technik gesehen werden, sind die anderen stärker auf der Seites des Geldes und der unternehmerischen Prozesse zu sehen.

\section{Duttenhofer und Daimler}

Bei Max von Duttenhofer fällt vor allem auf, dass er in den unterschiedlichsten Branchen $\mathrm{zu}$ finden ist ${ }^{27}$. Alles was neu war, schien ihn $\mathrm{zu}$ interessieren, wobei er sowohl dem Experimentieren als auch dem schnellen Umsetzen zugetan war. Duttenhofer und Daimler lernten sich über gemeinsame Bekannte wie z.B. Alfred

${ }^{26}$ Otto Schmid, 50 Jahre „Köln-Rottweil“. Aus der Geschichte der Fabrik Rottweil. Die Entstehungsgeschichte und die Leistungen eines bedeutenden Zweiges der chemischen Industrie, hg. von der I. G. Farbenindustrie Aktiengesellschaft Rottweil, Rottweil 1940, S. 62 .

27 Seine Aufsichtsratsposten werden im Nachruf in der Rottweiler Bürgerzeitung Nr. 184 vom 17.8.1903 aufgezählt: Er war Direktions-Präsident der Russischen Gesellschaft für Pulverfabrikation in St. Petersburg, er war Vorsitzender des Aufsichtsrats der Vereinigten Köln-Rottweiler Pulverfabriken, der Waffenfabrik Mauser in Oberndorf, der DaimlerMotorengesellschaft in Cannstatt, Vorsitzender des Kuratoriums der Centralstelle für wissenschaftlich-technische Untersuchungen, Neu-Babelsberg, Berlin. Er war stellvertretender Vorsitzender des Aufsichtsrats der Deutsch-Österreichischen Mannesmann Röhren-Werke in Düsseldorf, der Deutschen Röhrenwerke in Düsseldorf, der Motorfahrzeug- und Motorenfabrik A.G. in Marienfelde-Berlin, der Aktiengesellschaft für Feinmechanik vormals Jetter und Scherer in Tuttlingen, Mitglied des Aufsichtsrats und Komité-Mitglied der Deutschen Waffen- und Munitionsfabriken in Berlin, der Ludwig Loewe und Co, Aktien- 
Abb.6: Die Hohentwiel-Gesellschaft 1890. Max Duttenhofer ist halb liegend auf der Bank zu erkennen, stehend in der Mitte Gottlieb Daimler (Vorlage: Chronik der Hohentwiel-Gesellschaft, Stadtarchiv Rottweil).

[Die Abbildung kann aus rechtlichen Gründen online nicht bereitgestellt werden.]

Groß kennen und trafen sich informell bei Ausflügen auf den Hohentwiel ${ }^{28}$. Zunächst schien es einfach, Duttenhofer als Geldgeber zu gewinnen. Und dieser kannte mit Wilhelm Lorenz aus Karlsruhe einen weiteren Partner, der mit seinem Unternehmen schon so viel Geld verdient hatte, dass er als Investor auftreten konnte. Gottlieb Daimler war durch seine Abfindung aus der Gasmotorenfabrik Deutz und seine Einkünfte aus dem Motorenbau zwar wohlhabend, aber der Unterhalt seiner Firma und insbesondere der Entwicklungswerkstatt zehrte an seinem Vermögen und erlaubte auch keine Ausweitung des Unternehmens (Abb.6).

So wurde am 14. März 1890 ein Vertrag gemacht, der am 28. November notariell beglaubigt wurde und damit zur Gründung der Daimler Motorengesellschaft mit den drei Hauptaktionären Daimler, Lorenz und Duttenhofer führte. Nun war

gesellschaft in Berlin, von The Chilworth Gunpowder Company, Lmtd. in London, von The Nobel Dynamite-Trust Co., Lmtd. in London, der Dynamit-Aktiengesellschaft vormals Alfred Nobel und Co. in Hamburg, der Maschinenfabrik Eßlingen in Esslingen, von The British Mannesmann Tube Company, Lmtd. in London, der Hohenlohe'schen Nährmittelfabrik A.G. in Gerabronn, der Gesellschaft zur Förderung der Luftschiffahrt in Friedrichshafen, der Diskont-Gesellschaft in Berlin, der Württembergischen Metallwarenfabrik in Geislingen, der Westafrikanischen Pflanzungs-Gesellschaft „Bibundi“ in Hamburg, der Hanseatischen Kolonisations-Gesellschaft m.b.H. in Hamburg.

${ }^{28}$ Vgl. die Chronik der Hohentwiel-Gesellschaft im Stadtarchiv Rottweil. 
zwar zunächst Geld da, doch auch jede Menge Probleme. Schnell stellte sich heraus, dass man in der Geschäftspolitik nicht einig war, dass die beiden Finanziers Daimler überstimmen konnten und dass schließlich auch noch Qualitätsmängel, Patentstreitigkeiten, betriebliche Fehlentscheidungen und andere Schwierigkeiten den Betrieb in Bedrängnis brachten. Mit der Drohung der Insolvenz wurde Daimler 1894 aus dem Betrieb gedrängt, die Vereinsbank sprang mit weiteren Krediten und Duttenhofer und Lorenz mit weiteren Bürgschaften ein. 1895 kam es zwar zur Wiedervereinigung mit Daimler, doch das persönliche Verhältnis zu seinen Mitgesellschaftern war zerrüttet. Aus den Briefen Daimlers sind die Klagen überliefert, in welchen er Duttenhofer und Lorenz regelmäßig große Vorwürfe macht.

Daraus entstand auch in der Geschichtsschreibung das Bild eines gedemütigten Erfinders, der unter dem unnachgiebigen Druck und den Fehlentscheidungen seiner Kapitalgeber bis zu seinem Tod zu leiden hatte. Es spricht jedoch vieles dafür, dass auch Daimler von Anbeginn an unternehmerische Fehlentscheidungen gemacht hat und diese - gepaart mit seinen gesundheitlichen Problemen - zu einer grundsätzlichen Übellaunigkeit und einem nicht mehr zu behebenden Problem im Verhältnis zu seinen Mitgesellschaftern beitrug. Duttenhofer war sicher nicht zimperlich in seiner Art, doch erscheint er auch nicht nur als grober Geschäftsmann, sondern als Aufsichtsratsvorsitzender, der sich intensiv um die Entwicklung der Automobilindustrie bemühte. In einem Nachruf werden die Eigenschaften Duttenhofers zwischen zugewandt und rücksichtlos folgendermaßen beschrieben: Mebr aber noch als dies alles fesselte sein warmes hilfsbereites Herz und seine fröbliche, herzliche Art im Verkehr, die bei seinem oft rücksichtslosen Eingreifen in Verfolgung seiner stets uneigennützigen Pläne versöbnend wirkte und ibm in allen gerade denkenden Menschen rasch Freunde gewann. ${ }^{29}$

Daimler hatte schon nach Abschluss des Vertrages am 14. März 1890 Bedenken. Bis zur Beurkundung am 28. November 1890 führte er immerhin noch einen ergänzenden Syndikatsvertrag herbei, doch die grundsätzlichen Probleme waren damit nicht behoben. Seine Sacheinlage war zu gering bewertet worden, was von Anbeginn an zu Zwist führte. Auch seinem genialen Entwickler Wilhelm Maybach hatte er keine angemessene Position im neuen Unternehmen verschafft, so dass dieser es vorzog, in einem separaten Vertragsverhältnis zu Daimler zu bleiben. Erst im zweiten Anlauf, im Zuge des Wiedereintritts von Daimler in die DMG am 1. November 1895, erhielt auch Maybach die ihm angemessene Position des technischen Leiters sowie die Aktienanteile, die ihm aus seinen Vereinbarungen mit Daimler zustanden. Duttenhofer selbst hätte es genügt, wenn er nur Maybach für die DMG hätte gewinnen können, wie ein Abwerbeversuch 1895 belegt. Er hatte erkannt, dass die technische Entwicklung vor allem bei Maybach lag. Auch Harry Niemann resümiert, dass in diesen zehn Jahren Daimler nur etwa eineinhalb

${ }^{29}$ Nachruf der Centralstelle für wissenschaftlich-technische Untersuchungen von Wilhelm Will (Broschüre, Archiv der DaimlerChrysler AG, Akte Duttenhofer). 
Jahre lang direkt an der technischen Weiterentwicklung mitgearbeitet hat ${ }^{30}$. Sein Verdienst ist in dieser Zeit eher darin zu sehen, die Rahmenbedingungen für die Weiterentwicklung der Automobilproduktion zu schaffen. Erfolg hatte er dabei insbesondere in der Aufrechterhaltung und Durchsetzung einer Patentsituation, mit welcher wichtige Eckpfeiler für das Wirtschaften der Firma abgesichert werden konnten. Weniger Erfolg hatte er mit den Rahmenbedingungen für seine Firma, die ihn immer wieder in die Konfliktzone mit Duttenhofer führten. Von Anfang an waren die Ziele der Gesellschaft sowie deren Leitungsstruktur offenbar nicht gut geregelt. Während Duttenhofer beispielsweise nach den guten Erfahrungen der Monopolbildung aus dem Pulvergeschäft auch für die DMG eine Fusion mit der Deutzer Motorenfabrik anstrebte, wollte dies Daimler auf keinen Fall. Und die vermutlich beste Phase, in welcher er seinen Einfluss in der Firma entscheidend hätte verbessern können, ließ er ungenutzt verstreichen. Nachdem man schon viel gestritten hatte, boten Duttenhofer und Lorenz ihm in einem Nachtragsvertrag vom 26. Oktober 1892 die Übernahme von 102 Stück Aktien an.

Daimler hat diese Offerte nicht realisiert. Man kann daher nur spekulieren, dass sich die Situation nicht bis zum erzwungenen Ausscheiden Daimlers zugespitzt hätte, wenn er eine Aktienmehrheit erlangt hätte. Die persönliche Verfasstheit Daimlers hat sich danach, also auch nach dem Wiedereintritt in die Firma, offenbar nie mehr grundlegend verbessert. Selbst Maybach, der ihm immer loyal gegenüberstand, beklagt in einem Brief von 1896, dass Daimler nie mit einer Vereinbarung zufrieden war und konstatiert einen „krankhaften Zug“ im Verhalten Daimlers ${ }^{31}$. Maybach besprach daher viele Vorgänge eher mit Duttenhofer als mit Daimler. Auch von anderen Beteiligten wird Duttenhofer nicht als trickreicher Gegenspieler des Firmengründers wahrgenommen, sondern als weitsichtiger Unternehmer. So schreibt der kaufmännische Direktor Gustav Vischer zur Wiedervereinigung mit Daimler: „Bei all diesen Verhandlungen habe ich von neuem den großen, weiten Blick und die Geschäftskenntnis von Herrn Geheimrat Duttenhofer kennen und schätzen gelernt, ohne welchen eine Vereinbarung auf einer so gesunden Basis, wie sie nun erfolgt ist, überhaupt nicht möglich gewesen wäre“32.

Der Verbissenheit Daimlers stand offenbar eine gewisse Gelassenheit Duttenhofers gegenüber. Hauptgrund war sicherlich die vertragliche Schieflage zum Nachteil Daimlers, die bis zur Angst führte, dass sein Name auf der Liste der insolventen Unternehmen stehen könnte. Mit dieser Schmach hatten seine Mitgesellschafter ihm gedroht, während sie selbst einen hohen gesellschaftlichen Status erreicht hatten. Sicherlich waren aber auch die Handlungsoptionen und finanziellen Spielräume sehr unterschiedlich. So musste Daimler zwar keine Fusion mit der

30 Harry Niemann, Mythos Maybach, Stuttgart 2003, S. 133.

31 Ebd.

32 Ebd., S.119 (Brief Vischers an Wilhelm Dreuer). 
Deutzer Gasmotorenfabrik erleben, aber die Gründung eines Konkurrenzunternehmens.

Ganz parallel zum Rüstungsgeschäft wurde auch im Automobilgeschäft kurzerhand eine Neugründung in Berlin aufgezogen. Im Sommer 1897 wurde die Allgemeine Motorwagengesellschaft mbH gegründet, die ein Jahr später zur Motorfahrzeugs- und Motorenfabrik Berlin AG wurde und einerseits mit einem hohen Grundkapital von 2 Millionen Mark sowie mit Patenten aus Stuttgart ausgerüstet war. Die Geschäftsführung oblag formal der Deutschen Waffen- und Munitionsfabrik AG, die kurz zuvor mit der Ludwig Loewe AG zusammen entstanden war. Auch zur Gründung dieser Unternehmung standen also die bewährten Akteure aus der Finanz- und Rüstungsbranche bereit. Dass es sich auch um eine Umgehung von Daimler handelte, lässt die schnelle Fusion dieser Neugründung mit der Daimler-Motoren-Gesellschaft 1902 vermuten, also relativ bald nach dem Tod von Gottlieb Daimler.

Max Duttenhofer war in diesen ersten dreizehn Jahren mit kurzer Unterbrechung der Aufsichtsratsvorsitzende der Daimler-Motoren-Gesellschaft ${ }^{33}$. Er hatte nun Zugriff auf zwei Branchen. Das ließ sich durchaus gut verbinden, denn die Rüstungsbranche hatte traditionell gute Kontakte zu den Heeresverwaltungen und die Ausrüstung mit Militärfahrzeugen wurde natürlich schnell ein Geschäft. So gab Duttenhofer z. B. für das Maxim-Maschinengewehr, mit dessen Herstellung die Deutsche Waffen- und Munitionsfabrik 1897 begonnen hatte, in Cannstatt die Konstruktion eines passenden Fahrzeuges mit Maschinengewehr-Aufsatz in Auftrag. Diese spezielle Ausrüstung wurde für den Markt in Südafrika vorgesehen. In Südafrika war der Pulverkonzern schon seit 1893 mit einer eigenen Fabrik und einer Konzession für den Betrieb des Sprengstoff-Monopols aktiv ${ }^{34}$.

So lässt sich von den großen Strukturen bis in einzelne Kleinigkeiten eine hohe Dynamik beobachten, die von einer überschaubaren Anzahl von Akteuren in einem technisch und wirtschaftlich sehr innovativen Umfeld angestoßen wurde. Wie wichtig die Rüstungsindustrie im deutschen Südwesten war, ist heute jedoch kaum noch präsent. Die großen Hallenbauten der ehemaligen Waffen- und Munitionsfabriken in Karlsruhe (als IWKA bekannt) sowie die Kunststoffproduktion in dem Fabrikareal der ehemaligen Pulverfabrik in Rottweil haben eine Umnutzung erfahren. Nur Oberndorf ist bis heute mit der Waffenproduktion aufs engste verbunden.

${ }^{33}$ Duttenhofer übernahm von 1890 bis 1895 den Vorsitz des Aufsichtsrats, ab 1896 war er stellvertretendes Aufsichtsratsmitglied und 1900 bis 1903 wieder Aufsichtsratsvorsitzender.

34 Martin (wie Anm. 8) S.77. 